\title{
EVALUACIÓN DEL TREN INFERIOR ANTES DEL CURSO AVANZADO DE COMBATE (CAC) EN ALFÉRECES DE LA ESMIC
}

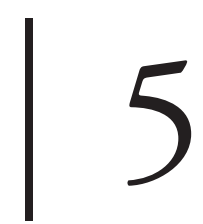

\author{
Perez Urrea Miguel Angel ${ }^{1}$ \\ Perlaza Daza Andres Felipe ${ }^{1}$ \\ Zuñiga Velasco Eulicer ${ }^{1}$ \\ Luz Marina Umbarila Espinosa
}

\section{Resumen}

Objetivo: Evaluar el tren inferior, con el test de fuerza antes del curso avanzado de combate (CAC) a los alféreces de la facultad de educación física de la Escuela Militar de Cadetes General "José María Córdova”

Materiales y métodos: Estudio observacional, descriptivo, de tipo cualitativo. Se realizó en la Escuela Militar de Cadetes General José María Córdova; Se constituye por un grupo de veintiocho (28) alféreces de la Facultad de Educación Física, los cuales realizan las pruebas iniciales o de base, antes del Curso de C.A.C. la selección de la población de forma aleatoria, mediante un muestreo no probabilístico, Resultados: Se analiza la base de datos constituida por 28 personas de los cuales 25 son hombres y 3 mujeres. Cada uno de ellos realizó un experimento en el cual se le solicitó que realizara un salto y con la ayuda del equipo OptoGait se obtuvieron los registros del tiempo de vuelo y la altura de vuelo de cada uno de los participantes realizando dos pruebas diferentes: CMJ y Squat Jump. Además, se tuvo en cuenta su índice masa corporal IMC; como dato fundamental, en donde los participantes del experimento cuentan con un peso promedio de $71 \mathrm{Kg}$ con una desviación de 9,47 Kg siendo el valor máximo $96 \mathrm{Kg}$ y el valor mínimo $52 \mathrm{Kg}$. La variable peso muestra una ligera tendencia de agrupamiento de los datos en valores menores a la media debido a que su coeficiente de asimetría es positivo: 0,52. Además el promedio de altura del grupo es de 172,67 cm con una desviación de 7,05 cm

1 Estudiantes de octavo nivel, grado alférez de la Facultad de Educación Física Militar. E-mail en orden de autoría: colmilperezurrea8@hotmail.com / perlaza_2001@hotmail.com / eumil_0519@hotmail.com

2 Asesora principal. Docente investigadora de la Facultad de Educación Física Militar. Magister en Ciencias y Tecnologías del Deporte y la Actividad Física. Grupo de investigación RENFIMIL. E-mail: Imarinaumbaes@hotmail.com 
con una altura máxima de $188 \mathrm{~cm}$ y mínima de $159 \mathrm{~cm}$. Los datos de la altura se encuentran agrupados en su mayoría en valores por debajo de la media, porque su coeficiente de asimetría es de 0,25. Conclusiones: Después de las pruebas se llega a la conclusión de que todos los alféreces tienen buena potencia en el tren inferior al momento de presentar los test lo cual daría el tener buenos resultados al momento de estar en el curso avanzado de combate realizando pruebas que requieran un gran esfuerzo en las piernas y resistencia en este tren.

Palabras clave: Fuerza potencia, curso avanzando de combate, población militar en formación.

\section{Introducción}

Los test de potencia son frecuentemente utilizados para evaluar la musculatura extensora de las extremidades inferiores. Especialmente en aquellos deportes que involucren saltos o cambios rápidos de posición (Vandewalle H, et.al 1989).

El desarrollo de la musculatura es un elemento esencial en el rendimiento de muchas actividades dentro del contexto Militar, sustentada por los alférez de la Escuela Militar de VIII nivel que han vivido las diferentes pruebas físicas y/o cursos que a través de su carrera han implementado, por esta razón se debe dar importancia a la fuerza máxima en el tren inferior, uno de los eventos donde la exigencia física se pone a prueba es para la realización del Curso Avanzado de combate(CAC) (Anexo1.); donde las jornadas son de extremas condiciones física y mentales, lo cual nos da a conocer la necesidad de implementar trabajos para fortalecimiento del tren inferior.

Este documento contiene los antecedentes, metodología y diferentes aspectos los cuales nos guiaran y nos ayudaran a comprender mejor el trabajo realizado en esta tesis de grado, teniendo en cuenta el seguimiento llevado a los participantes de los diferentes test de evaluación, aplicados y sus conclusiones al comparar los resultados arrojados por dichos test.1. Revisión documentos sobre los test Squad Jump y Counter Movement Jump; 2. La metodológica sobre la aplicación de los test. 3. Análisis y resultados de los datos. 4. Conclusiones.

En el desarrollo de las actividades militares se ejerce la aplicación de diversas fuerzas, sobre los segmentos corporales correspondientes, de manera que, si no se produjese esta aplicación no existiría actividad física. La valoración de esta capacidad de producir los distintos tipos de fuerzas, mediante muy diversos métodos, es una materia que ha ocupado a la medicina del deporte desde hace mucho tiempo. El entrenamiento de la potencia se ha convertido en una herramienta fundamental 
a la hora de optimizar el rendimiento, principalmente en los deportes y/o donde la fuerza explosiva y la velocidad de movimiento son determinantes (Naclerio, 2001)

En términos de velocidad y tiempo se dice que estas características deben ser consideradas al realizar los ejercicios de preparación física, donde, todavía no está bien determinado en qué medida el control de la fuerza, velocidad y potencia producida en cada gesto influyen en la eficiencia para mejorar las acciones propias del deporte y o de la actividad física. (Baker y Nance 1999; Biscioti 2001).

En la comunidad de la Escuela Militar de Cadetes General "José María Córdova”, es de notar la diferencia de actividad física que realizan los cadetes de las Facultad de Educación Física Militar, que para este caso son los participantes evaluados estudiantes de séptimo nivel, quienes dentro de su pensum académico, ven áreas donde desarrollar sus capacidades y habilidades básicas, factor que hace la diferencia con las demás carreras completarías, esto ha permitido que a lo largo de los diferentes cursos, a los alférez de Educación Física Militar sobresalir en la mayoría de pruebas físicas, lo que no queda muy claro son las consecuencias del después de Curso Avanzado de Combate, no hay evidencias de los cambios en su tren inferior o si hay alguna disminución en su potencia.

Se hace claridad que por cuestión de tiempo se realiza la investigación antes de que los alférez se desplacen a Tolemaida, sitio donde se llevara a cabo dicho curso; el proyecto queda abierto para que otro grupo de la Facultad realice la toma de datos nuevamente con los test que se utilizaron en la metodología, para el momento de la llegada y poder entregar en un futuro proyecto de grado datos concluyentes de si hubo cambio o no luego del tiempo y condiciones establecidas para dicho entrenamiento especializado.

El Curso Avanzado de Combate es un programa de instrucción y entrenamiento, donde los alumnos de la Escuela Militar de Cadetes "General José María Córdova” tienen como objetivo entrenarse y certificarse en las áreas técnica, táctica, humanística y física para que adquieran los conocimientos básicos para liderar un pelotón en el desarrollo de operaciones militares como requisito indispensable para su ascenso teniendo en cuenta que al momento de ascender su resistencia ,potencia , y fuerza muscular debe ser la adecuada para liderar en sus futuras unidades. (ESERT, 2006)

Poder realizar este Curso en el grado de alférez, para los participantes significa adquirir experiencia para su posterior fase de mando y así poder orientar a quienes estarán bajo sus órdenes entiendan todo el significado de la guerra irregular, a través de su experiencia vivida; Además, ya para su carrera como oficiales, 
poder tomar las mejores decisiones cuando lideren pequeñas unidades al inicio de su carrera profesional y aún más, cuando con el pasar del tiempo lleven la batuta de tropas enteras. (Escuela Militar de Cadetes, 2007)

La actividad diaria inicia todos los días a las 04:15 am para el trote regular que hace parte de la vida de cualquier militar, pero aún más, se enfocaron en prepararse para las certificaciones que se aproximan de 5,7 y 10 kilómetros respetivamente. (Escuela Militar de Cadetes, 2007)

Entre los ejercicios realizados, los alumnos recibirán instrucción de planeamiento, operaciones de combate irregular, SOMT (señalador de objetivos militares) instrucción que la desarrolla y certifica personal de oficiales de la fuerza aérea del Comando de Combate aéreo No 4, geografía militar, graficación militar, polígonos de fusil Galil, polígonos en movimiento y de reacción, polígonos de ametralladora M-60 y pistola. Así mismo, disparo de mortero de $81 \mathrm{~mm}$, de MGL y lanzamiento de granada de mano. (Escuela Militar de Cadetes, 2007)

Considerando la Declaración de Helsinki y la Resolución 008439 de 1993 del Ministerio de Salud para la investigación en seres humanos, donde se siguieron todos los protocolos éticos establecidos, en especial en lo referente a que el estudio no afectaría la salud de los participantes.

\section{Organigrama cursos de combate}

La Jefatura de Educación y Doctrina del Ejército Nacional cuenta en su organigrama (Fig.1) con la Dirección de Instrucción, Entrenamiento y Reentrenamiento y en ella una subestructura denominada Subdirección de Reentrenamiento que establece las características que deben poseer los Cursos de Combate.

\section{Materiales y métodos}

Tipo de estudio: Se realizó un estudio observacional, descriptivo, de tipo cualitativo.

Universo: Escuela Militar de Cadetes General José María Córdova

Población: Facultad de Educación Física Militar

Muestra: Se constituye por un grupo de veintiocho (28) alféreces de VII nivel de la Escuela Militar de Cadetes, matriculados en la Facultad de Educación Física Militar, los cuales realizan las pruebas iniciales o de base, antes del Curso de C.A.C. 


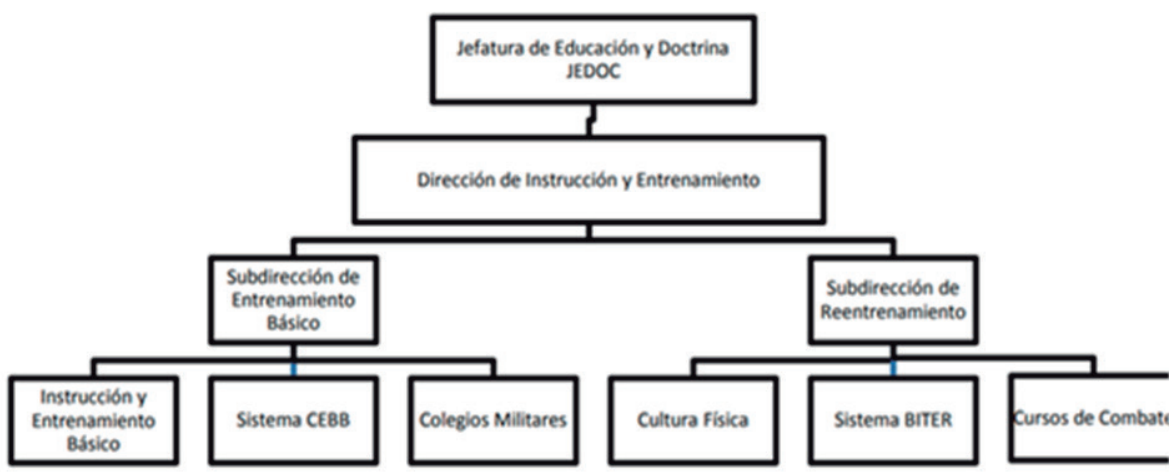

Figura1. Organigrama JEDOC, modificado por los autores Fuente: ejército.mil.co, (s.f.)

Tamaño de la Muestra: Se realizó la selección de la población de forma aleatoria, mediante un muestreo no probabilístico, en el cual se seleccionaron Veintiocho (28) alféreces pertenecientes a Séptimo (7) Nivel de la Facultad de Educación Física Militar.

Criterios de inclusión

- Que sean alféreces 18 y los 23 años pertenecientes a la Facultad de Educación Física Militar

- Que estén próximos a asistir en el segundo semestre del año al curso avanzado de combate

Criterios de Exclusión

- Que posean alguna lesión de miembro inferiores

- Alféreces que presenten algún tipo de enfermedad que les impida la realización de los test.

\section{Resultados}

De acuerdo al análisis estadístico descriptivo y la valoración de la composición corporal, se obtuvo los siguientes resultados:

Se analiza la base de datos constituida por 28 personas de los cuales 25 son hombres y 3 mujeres. Cada uno de ellos realizó un experimento en el cual se le solicitó que realizara un salto y con la ayuda del equipo OptoGait se obtuvieron los registros del tiempo de vuelo y la altura de vuelo de cada uno de los participantes realizando dos pruebas diferentes: CMJ y Squat Jump. 
Además, se tuvo en cuenta su índice masa corporal IMC; como dato fundamental, en donde los participantes del experimento cuentan con un peso promedio de $71 \mathrm{Kg}$ con una desviación de 9,47 Kg siendo el valor máximo $96 \mathrm{Kg}$ y el valor mínimo $52 \mathrm{Kg}$. La variable peso muestra una ligera tendencia de agrupamiento de los datos en valores menores a la media debido a que su coeficiente de asimetría es positivo: 0,52 . Además el promedio de altura del grupo es de $172,67 \mathrm{~cm}$ con una desviación de 7,05 cm con una altura máxima de $188 \mathrm{~cm}$ y mínima de $159 \mathrm{~cm}$. Los datos de la altura se encuentran agrupados en su mayoría en valores por debajo de la media, porque su coeficiente de asimetría es de 0,25.

El análisis se divide en cada una de las pruebas para posteriormente hacer una comparación de resultados.

\section{Resultados prueba CMJ}

Las variables medidas además del peso y la altura corresponden al tiempo de vuelo y a la altura registrada en el salto. En general, el tiempo de vuelo promedio es de 0,47 s con una desviación de 0,05 s; el tiempo de vuelo más largo es de 0,54 $s$ mientras que el más corto es de $0,37 \mathrm{~s}$. Los datos del tiempo de vuelo se agrupan por encima del valor medio con un coeficiente negativo de - 0,70 . El promedio de la altura del salto es de $28 \mathrm{~cm}$ con una desviación de 6,04 cm con el valor máximo en $36,4 \mathrm{~cm}$ y el mínimo en 15,6. Los datos se agrupan en valores ligeramente mayores a la media con un coeficiente de asimetría negativo de $-0,50$.

Se realizó una correlación entre las variables: peso, altura, tiempo de vuelo y altura de vuelo para verificar los coeficientes de correlación entre ellas. Los resultados se observan en la figura 2.

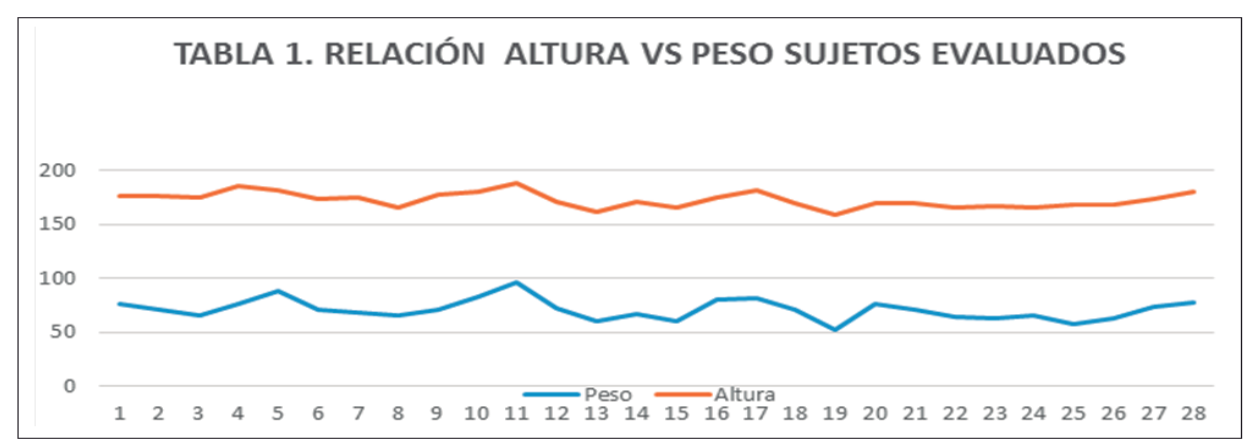

Figura 2. Relación altura vs peso sujetos evaluados Fuente: Original de los autores 
Tabla 1. Correlación entre las variables de la prueba CMJ

\begin{tabular}{|c|c|c|c|c|}
\hline & Peso & Altura & TVuelo[s] & Altura $[\mathrm{cm}]$ \\
\hline Peso & 1 & & & \\
\hline Altura & 0,86073099 & 1 & & \\
\hline TVuelo[s] & 0,15858107 & 0,15314802 & 1 & \\
\hline Altura $[\mathbf{c m}]$ & 0,1755687 & 0,16157792 & 0,99816955 & 1 \\
\hline
\end{tabular}

Fuente: Original de los autores

Como se observa en la (figura 2), el coeficiente de correlación entre el peso y la altura es de 0,86 , lo cual indica que existe una alta relación entre estas dos variables. Adicionalmente, se observa que no existe relación alguna entre el peso del participante y el tiempo de vuelo, el peso del participante y la altura de vuelo ni entre la altura del participante y el tiempo de vuelo ni la altura del participante y la altura de vuelo. Sin embargo, como es de esperarse, se presenta una alta relación entre el tiempo de vuelo y la altura de vuelo. El coeficiente de correlación entre estas dos variables es de 0,99 por lo cual se puede concluir definitivamente que entre más tiempo de vuelo mayor altura en el salto.

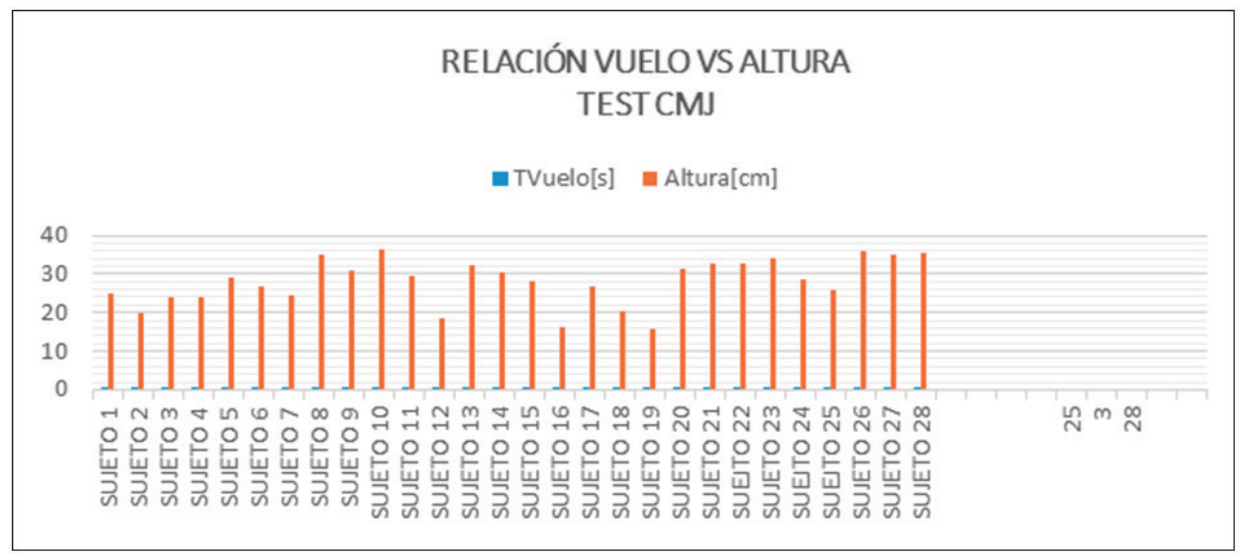

Gráfica 3. Correlación entre las variables de tiempo de vuelo y altura test CMJ Fuente: Original de los autores 


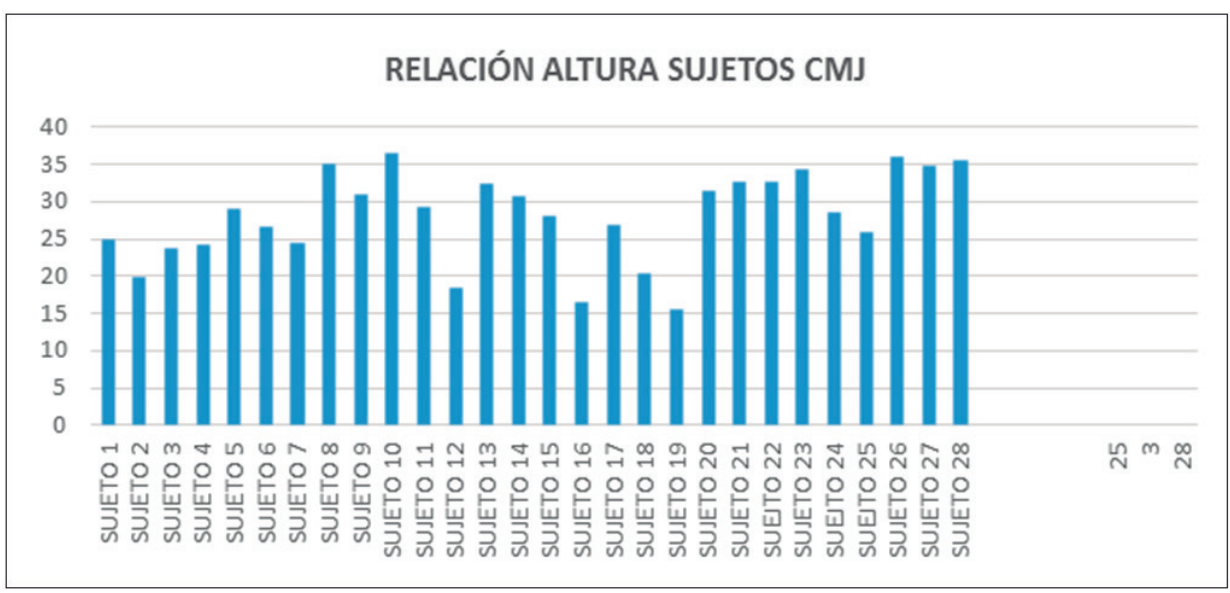

Gráfica 4. Correlación entre la altura y el sujeto CMJ

Fuente: Original de los autores

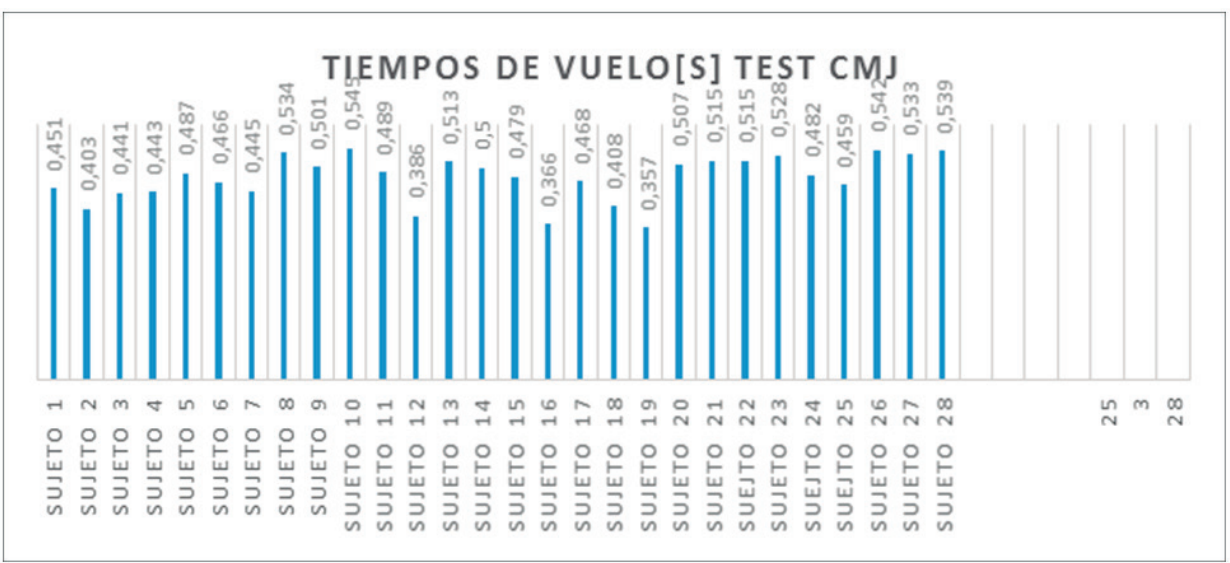

Gráfica 5. Tiempos de Vuelo Test CMJ

Fuente: Original de los autores

\section{Resultados prueba Squat Jump}

En cuanto a los datos obtenidos con la prueba Squat Jump se obtienen los resultados promedio del tiempo de vuelo que es $0,45 \mathrm{~s}$ con una desviación de 0,04 s y un coeficiente de asimetría de - 0,54 lo que indica que los datos se encuentran agrupados en valores ligeramente mayores a la media. El tiempo de vuelo menor es de 0,32 s y el mayor 0,53 . En cuanto a la altura de salto, se observa que su promedio es de $25,18 \mathrm{~cm}$ con una desviación de $5,37 \mathrm{~cm}$ y que los datos se encuentran agrupados siendo mayores a la media con un coeficiente de asimetría de $-0,23$. La mayor altura de salto alcanzada es de $34,8 \mathrm{~cm}$ mientras que la menor de $12,8 \mathrm{~cm}$. 


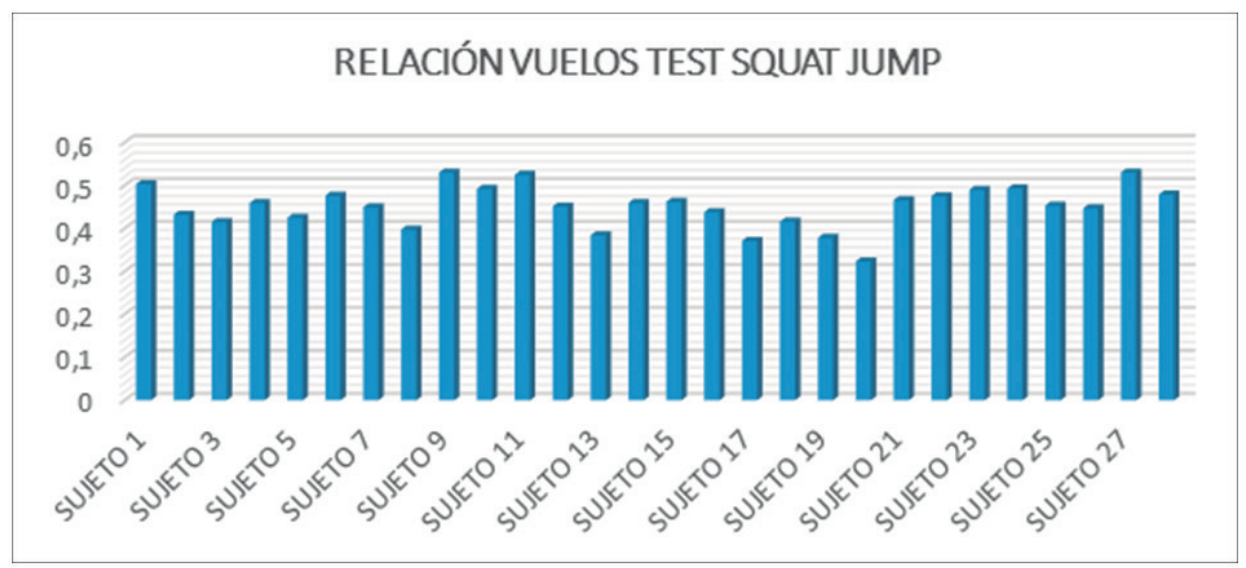

Gráfica 6. Relación Vuelos Test Squat Jump Fuente: Original de los autores

La tabla de correlaciones entre las variables se muestra en la Tabla 2.

Tabla 2. Correlación entre las variables de la prueba Squat Jump

\begin{tabular}{|c|c|c|c|c|}
\hline & Peso & Altura & TVuelo[s] & Altura $[\mathbf{c m}]$ \\
\hline Peso & 1 & & & \\
\hline Altura & 0,86073099 & 1 & & \\
\hline TVuelo[s] & 0,22071476 & 0,32085052 & 1 & \\
\hline Altura $[\mathrm{cm}]$ & 0,23986133 & 0,32800986 & 0,99721976 & 1 \\
\hline
\end{tabular}

Fuente: Original de los autores

Como se muestra en la tabla y de la misma manera que en la prueba de salto anterior, las correlaciones fuertes se encuentran entre las variables peso y altura con un coeficiente de 0,86 y entre el tiempo de vuelo y la altura de salto con un coeficiente de 0,99 . Se concluye entonces que las variables de peso y altura con relación al tiempo de vuelo y la altura de salto no se relacionan estadísticamente.

Haciendo una comparación descriptiva entre las pruebas de salto se concluye que:

El promedio del tiempo de vuelo en la prueba Squat Jump es mayor que en la prueba CMJ, sin embargo, es de resaltar que la desviación de los datos constituye una gran diferencia debido a que se muestra que los datos de la prueba Squat Jump se encuentran mucho más dispersos que en la prueba CMJ. 


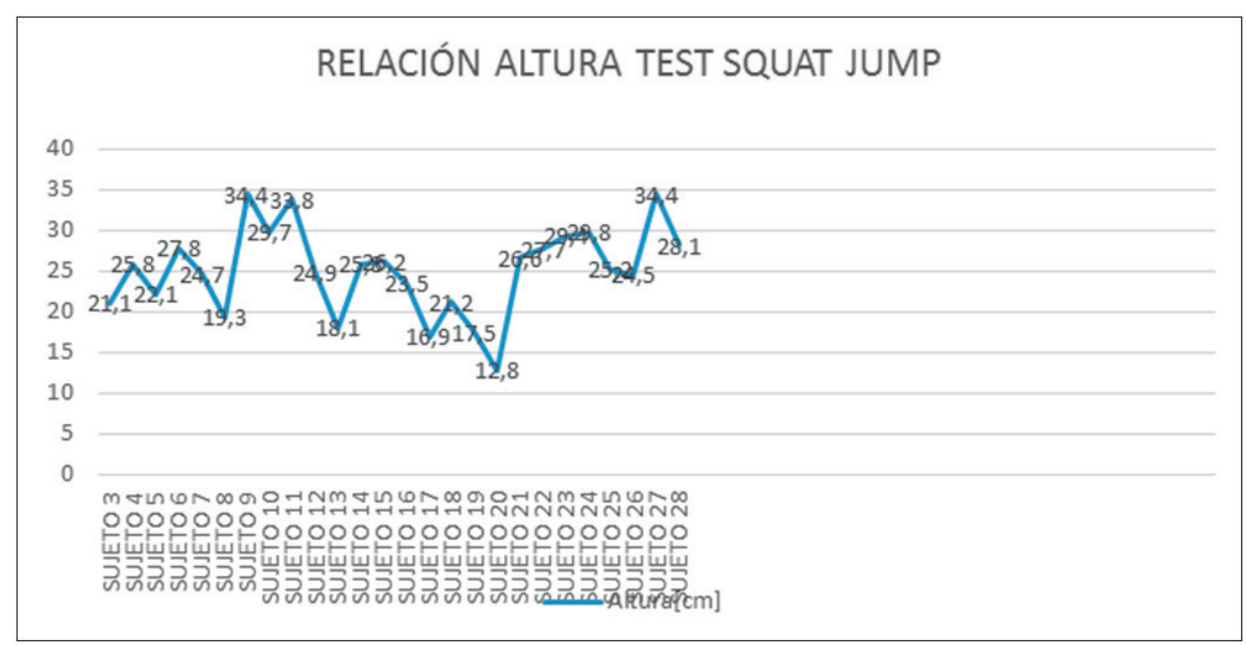

Gráfica 7. Relación Altura Test Squat Jump Fuente: Original de los autores

\section{Discusión}

Hacemos un relación con base a los baremos encontrados de un estudio realizado en deportistas de atletismo en promedio estimado de edad similar al de los alféreces de la facultad de educación física de la escuela militar donde vemos que los dos test realizado a los alféreces se encuentran por encima de la media con base a las diferentes tablas de datos por lo cual podemos decir que los alféreces poseen una buena potencia en el tren inferior aunque se puede y debe incrementar por medio de un entrenamiento adecuado al mismo curso avanzado de combate.

Tabla 3. Valoración Test SJ, CMJ

\begin{tabular}{|c|c|c|c|c|c|c|}
\hline \multirow[t]{2}{*}{ TABLA 1} & \multicolumn{2}{|c|}{ GLOBAL } & \multicolumn{2}{|c|}{ VARONES } & \multicolumn{2}{|c|}{ MUJERES } \\
\hline & MEDIA & DES EST & MEDIA & DES EST & MEDIA & DES EST \\
\hline S. & 33.00 & 6.41 & 34.49 & 5.13 & 26.31 & 4.47 \\
\hline $\mathrm{CMJ}$ & 36.58 & 8.55 & 39.23 & 5.58 & 29.47 & 10.86 \\
\hline
\end{tabular}

Fuente: Original de los autores 


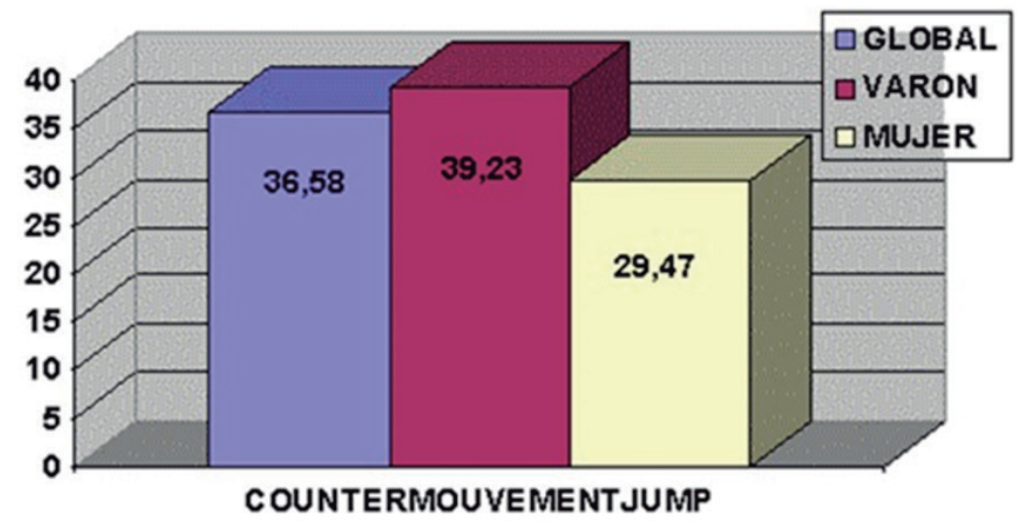

Gráfica 8. Countermouvement Jump $\mathrm{F}_{1}$

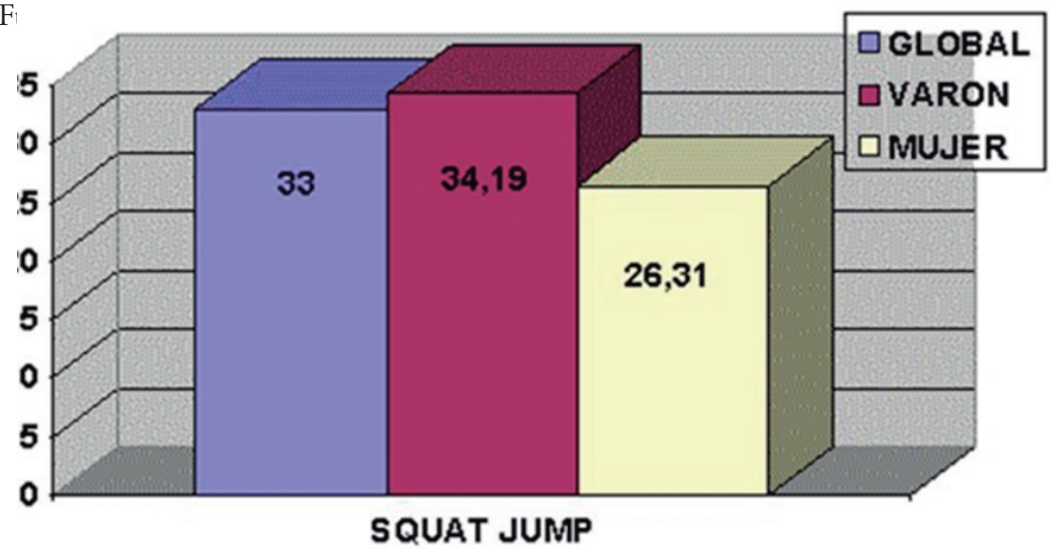

Gráfica 9. Squat Jump

Fuente: Original de los autores

\section{Conclusiones}

- En cuanto a los máximos y mínimos se cuentan con datos similares en cuanto al tiempo de vuelo. Mientras que en la altura de salto si se registra una diferencia significativa en centímetros.

- Como se observa el coeficiente de correlación entre el peso y la altura es de 0,86 , lo cual indica que existe una alta relación entre estas dos variables. 
- Se recomienda que se tomaran mayor cantidad de datos y que la muestra tuviera una cantidad diferencial de hombres y mujeres para correlacionar los datos con género, correlación que no se puede realizar en el presente estudio porque solamente 3 mujeres participaron en el experimento y los datos se sesgarían hacia el género masculino.

- Se recomienda realizar planes de entrenamiento para llegar a un fortalecimiento de articulaciones tendones y músculo del tren inferior con anticipación al curso avanzado de combate con el fin de prevenir lesiones futuras.

- Se recomienda planes de entrenamiento tales como el fortalecimiento del tren inferior por medio de diferentes ejercicios y entrenamiento, en el gimnasio de la Escuela Militar con los entrenadores personales con los que cuenta el gimnasio, realizar un entrenamiento específico del grupo muscular y articular del tren inferior de cada alférez próximo a realizar el curso avanzado de combate.

\section{Referencias}

Alepuz, J. R. (2014). instituto de medicina del sueño dormir mejor es salud. Obtenido de www. dormirbien.info

Bellido, D. (2006). Índice de masa corporal. de Luis D.

Bosco C. Test de Bosco. In: Paidotribo E, editor. La valoración de la fuerza con el test de Bosco. Barcelona: Hurope S.L.; 1994. p. 35-137

ejercito.mil.co, (s.f.). [Imagen]. Recuperado de http://www.ejercito.mil.co/recursos_user/imagenes// editores/163207/organigrama_dir_instruc_01.jpg

Escuela Militar de Cadetes. (2007). Curso avanzado de combate. Bogotá.

ESERT. (2006). Pensum curso avanzado de combate. Tolemaida.

FÍSICA, I. C.-D. (octubre de 2010). Fundamentos biológicos y bases del entrenamiento deportivo. Obtenido de https://efiesconselleria.files.wordpress.com/2010/09/composicion-corporal.pdf

Harman EA, Rosenstein MT, Frykman PN, Rosenstein RM (1990). The effect of arms and countermovement on vertical jumping. Med Sci Sports and Exerc 22:825-33

Higuera, D. J. (2008). www.psicoterapeutas.com. Obtenido de http://www.psicoterapeutas.com/ pacientes/suenyos.htm

Keys, G. y. (1987). manual de nutrición y dietética. Obtenido de https://www.ucm.es/data/cont/ docs/458-2013-07-24-cap-2-composicion-corporal55.pdf

Masse, p. 1. (2000). Aplicación del test de salto CMJ (Counter Movement Jumpo) con la plataforma de contacto. Aplicación del test de salto CMJ (Counter Movement Jumpo) con la plataforma de contacto. 
Masse, P. L. (2001). Aplicación del Test de Salto CMJ (Counter Movement Jump) con Plataforma de Contacto. Blogs Evaluación.

Mott, J. W. (1999). entrenamiento físico.

Naclerio A. F. (2001) Entrenamiento de la fuerza con pesas: cómo determinar la intensidad del esfuerzo y los diferentes tipos de fuerza a entrenar. http:// www.efdeportes.com/ Revista Digital - Buenos Aires - Año 6 - No 29

Ramón, J. (2009). ARCHIVOS DE MEDICINA DEL DEPORTE. Obtenido de http://www. femede.es/documentos/ConsensoCine131.pdf

Sapega AA, Nicholas JA, Sokolow D, Saranti A (1982). The nature of torque overshoot in Cybex isokinetic dynamometry. Med Sci Sports and Exerc 14(5):368-75

Vallordo, E. (2012). El Squat Jump. entrenamiento deportivo, 1,2,3. Vandewalle H, Péres G, Sourabié B, Stouvenel O, Monod H. Force-velocity relationship and maximal anaerobic power during crancking exercise in young swimmers. Int J Sports Med 1989; 10: 439-45.

Verkhoshansky, y. (1996). Componenti e Structura Dell impegno explosivo di Forza. Rivista di cultura Sportiva, no 34, 15-21.

Williams, D. T. (2007). Masa grasa. 